\title{
Política de drogas ilegales en España: la opinión de los profesionales del ámbito sanitario y del legal
}

\section{Illicit drug policy in Spain: the opinion of health and legal professionals}

\author{
Paola Rossi*, Ester Blay**, Victor Costela***, Marta Torrens*,**** \\ * Institut de Neuropsiquiatria i Addiccions, Hospital del Mar, IMIM (Institut Hospital del Mar d'Investigacions Mèdiques), \\ Barcelona. \\ ** Departament de Dret Públic, Universitat de Girona. \\ *** Universitat de Granada. \\ **** Departament de Psiquiatria, Universitat Autònoma de Barcelona.
}

\section{Resumen}

La elevada frecuencia de conductas delictivas y problemas legales relacionados con las adicciones a sustancias genera un terreno de interacción entre los ámbitos legal y sanitario. En este contexto se ha llevado a cabo un estudio multicéntrico de las opiniones de los profesionales tanto del ámbito legal como del sanitario sobre la legislación relacionada con las drogas y su implementación en la práctica de acuerdo al marco legal vigente.

Se administró a 230 profesionales tanto del ámbito legal como del sanitario de Barcelona, Granada y Bilbao un cuestionario de respuesta múltiple diseñado ad hoc, con datos sociodemográficos y laborales y preguntas para valorar la opinión de los encuestados sobre la respuesta a la delincuencia relacionada con drogas y su postura en relación con la política en materia de drogas.

Los principales resultados mostraron que ambos grupos de profesionales valoran las medidas penales alternativas (MPA) como herramientas útiles para prevenir los delitos relacionados al consumo, apostando por la ampliación de su aplicación. También coinciden en valorar positivamente la actual regulación del consumo de cannabis. Los profesionales del ámbito sanitario muestran una actitud más permisiva de cara a la aplicación de MPA, pero ambos grupos reconocen oportuno endurecer la sanción en caso de reincidencia delictiva. Los dos grupos muestran una satisfacción relativa con el sistema de atención a las adicciones en los aspectos estudiados y expresan insatisfacción con las políticas actuales sobre drogas.

Palabras clave: Adicción, responsabilidad penal, legislación sobre drogas, despenalización, sistema sanitario.

\begin{abstract}
The high frequency of criminal behaviour and related legal problems associated with substance addiction generates a field of interaction between legal and healthcare systems.

This study was developed as a multicentre project to investigate the opinions of professionals from legal and healthcare systems about policies on illegal drugs and their implementation in practice. A multiple choice questionnaire designed ad hoc was administered to a sample of 230 professionals from legal and healthcare fields working in the cities of Barcelona, Granada and Bilbao. The questionnaire included sociodemographic and work-related data, and assessed interviewees' information about the response to drug-related crime and opinion on drug policy issues. This article presents the results from Spain.

The main results showed that both groups of professionals value alternative measures to imprisonment (AMI) as useful tools to prevent offenses related to drug use and claim a broader application of AMI. They also evaluated positively the regulations on cannabis use in effect. Though the attitude of healthcare professionals towards the application of AMI is more permissive, both groups favour restricting these sanctions in cases of recidivism. Both groups show mild satisfaction with the current addiction healthcare system and express dissatisfaction with actual drug policies in Spain.

Keywords: Addiction, criminal liability, drug policies, decriminalizing, healthcare system.
\end{abstract}




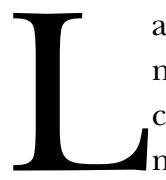

as adicciones a sustancias y su tratamiento suponen un reto para los profesionales, tanto por la complejidad y gravedad de sus características clínicas como por su asociación con problemas sociales y legales secundarios a su consumo. La delincuencia relacionada con el consumo de sustancias durante mucho tiempo ha sido la base del estigma de las adicciones. La elevada frecuencia de conductas delictivas y problemas legales relacionados con ésta en pacientes con trastorno adictivo genera un terreno de interacción entre el ámbito legal y el de la atención sanitaria (Esbec y Echeburúa, 2016). El conocimiento actual en el campo de las adicciones permite definir sin ambigüedades cierta conducta delictiva como una consecuencia de una patología más compleja, por lo que hay que avanzar en el conocimiento y en el abordaje de una problemática tan relevante a nivel de costes sociales y económicos (European Monitoring Centre for Drugs and Drug Addiction, 2007).

En el seno de la Unión Europea no existe una uniformidad jurídica entre los diferentes países en relación al consumo de sustancias ilegales (European Monitoring Centre for Drugs and Drug Addiction, 2015). Estados como Polonia mantienen una penalización del consumo de sustancias, así que el paciente con trastorno por dependencia de sustancia es autor de un delito en el momento en que consume. En España, como veremos más adelante, se escinde el acto delictivo relacionado con la producción y distribución de la sustancia de abuso del consumo personal y la tenencia de droga para el autoconsumo, que no están penalizados. Esta diferencia repercute en las prácticas de los profesionales implicados tanto a nivel legal como a nivel de seguimiento médico, y es reflejada en la valoración que los profesionales hacen del mismo.

\section{Breve referencia al marco jurídico en España: regulación y datos disponibles}

A diferencia de lo que sucede en otras jurisdicciones europeas y americanas, la opción político criminal en el ordenamiento español ha consistido en no castigar penalmente el autoconsumo, ni la posesión de pequeñas cantidades para el mismo, mientras sí se castigan penalmente las conductas de cultivo, elaboración o tráfico ilegal de drogas tóxicas, estupefacientes o sustancias psicotrópicas (Capítulo III De los delitos contra la salud pública, del Título XVII De los delitos contra la seguridad colectiva, del Código Penal aprobado por Ley Orgánica 10/1993, de 23 de noviembre). El derecho administrativo, sin embargo, sí sanciona la tenencia ilícita y el consumo de drogas tóxicas, estupefacientes o sustancias psicotrópicas en espacios públicos (Ley Orgánica 4/2015, de 30 de marzo, de protección de la seguridad ciudadana).

El Derecho penal, además, recoge respuestas sancionadoras diferenciadas y específicas cuando la persona que ha cometido un delito lo ha realizado bajo los efectos de las drogas o por causa de su adicción a las mismas (Anexo I). Como consecuencia de la aplicación de esta normativa, las personas que, habiendo sido condenadas por la comisión de un delito y cuyo problema con las drogas ha sido detectado antes de la condena y recogido en la sentencia como eximente o atenuante, pueden encontrarse, en vez de en prisión, en una de las siguientes situaciones: medida de seguridad privativa de libertad consistente en un internamiento en un centro de deshabituación, medida de seguridad no privativa de libertad consistente en un tratamiento ambulatorio en un contexto de libertad vigilada o suspensión de la pena de prisión para drogodependientes. Este conjunto de respuestas se incluye en el concepto más amplio de Medidas Penales Alternativas (MPA) a la prisión. En España, en el año 2013 los Servicios de Gestión de Penas y Medidas Alternativas gestionaron 24.865 sentencias de MPA que se correspondían con suspensiones y sustituciones de condena. El 58\% fueron por delitos de violencia de género, el $5 \%$ por delitos de seguridad vial y el $37 \%$ por otros delitos, entre los que se incluyeron los relacionados con consumo de sustancias adictivas (Delegación del Gobierno para el Plan Nacional sobre Drogas, 2013).

Puede suceder, finalmente, que la problemática relacionada con la adicción no se detecte o no se aborde a nivel judicial en la imposición de la pena, y que una persona que ha cometido la infracción a causa de su dependencia o que presente problemas de adicción ingrese en prisión. En estos casos, el sistema penitenciario ofrece diferentes alternativas de prevención, reducción de daños y tratamiento (anexo I). En 2014, 4.783 personas condenadas a prisión fueron incluidas en programas de tratamiento de adicción a sustancias en el contexto de la libertad condicional y el tercer grado penitenciario (Secretaría General de Instituciones Penitenciarias, 2014).

La existencia de respuestas penales distintas a la prisión y sensibles a la problemática de las adicciones a sustancias significa que hay personas que por mandato de una resolución judicial están cumpliendo una pena o una medida de seguridad cuyo contenido esencial es un tratamiento de deshabituación fuera de un centro penitenciario. Esto comporta la necesidad de que agencias y profesionales de distintos ámbitos, el judicial, el de la ejecución penal, el social, el educativo y el sanitario se coordinen para aplicar lo que en resumen constituye a la vez un mandato judicial y un tratamiento médico. Ello implica la existencia de un técnico, generalmente un psicólogo o un trabajador social, dependiente de Secretaría General de Instituciones Penitenciarias o del Departamento de Justicia en el caso de Catalunya, que es responsable de la ejecución de la resolución judicial, y a través de su contacto con los distintos profesionales sanitarios responsables, informa al juez sobre el grado de cumplimiento del mismo. 
Anexo I. Cuadro resumen de la normativa penal y penitenciaria específica para personas con dependencia de sustancias tóxicas.

\begin{tabular}{|c|c|c|c|}
\hline & Institución & Circunstancia de aplicación & Disposición normativa \\
\hline \multirow[t]{3}{*}{$\begin{array}{l}\text { Determinación de } \\
\text { la responsabilidad } \\
\text { penal }\end{array}$} & $\begin{array}{l}\text { Causa de exención de la } \\
\text { responsabilidad penal }\end{array}$ & $\begin{array}{l}\text { Intoxicación plena o síndrome de abstinencia en } \\
\text { el momento de delinquir, siempre que impidan } \\
\text { comprender ilicitud del hecho y actuar conforme esa } \\
\text { comprensión }\end{array}$ & Art. $20.2^{\circ} \mathrm{CP}$ \\
\hline & $\begin{array}{l}\text { Causa de exención incompleta de } \\
\text { la responsabilidad penal }\end{array}$ & $\begin{array}{l}\text { Intoxicación plena o síndrome de abstinencia en el } \\
\text { momento de delinquir, a falta de requisitos para la } \\
\text { exención completa }\end{array}$ & $\begin{array}{l}\text { Art. } 21.1^{\circ} \text { en relación con art. } \\
20.2^{\circ} \mathrm{CP}\end{array}$ \\
\hline & $\begin{array}{l}\text { Circunstancia atenuante de la } \\
\text { responsabilidad penal }\end{array}$ & $\begin{array}{l}\text { Actuación a causa de la grave adicción a sustancias } \\
\text { tóxicas }\end{array}$ & Art. $21.2^{\circ} \mathrm{CP}$ \\
\hline \multirow[t]{3}{*}{$\begin{array}{l}\text { Respuestas } \\
\text { sancionadoras } \\
\text { específicas }\end{array}$} & $\begin{array}{l}\text { Medida de seguridad de } \\
\text { internamiento en centro de } \\
\text { deshabituación }\end{array}$ & $\begin{array}{l}\text { Personas exentas total o parcialmente de } \\
\text { responsabilidad por art. } 20.2^{\circ} \text { o } 21.1^{\circ} \mathrm{CP}\end{array}$ & Art. $102.1^{\circ}$ y $104 \mathrm{CP}$ \\
\hline & $\begin{array}{l}\text { Medida de seguridad de libertad } \\
\text { vigilada con obligación de } \\
\text { tratamiento de deshabituación } \\
\text { ambulatorio }\end{array}$ & $\begin{array}{l}\text { Personas exentas total o parcialmente de } \\
\text { responsabilidad por art. } 20.2^{\circ} \text { o } 21.1^{\circ}\end{array}$ & Art. 106.1.k CP \\
\hline & $\begin{array}{l}\text { Suspensión de la pena de prisión } \\
\text { con obligación de seguir un } \\
\text { tratamiento de deshabituación }\end{array}$ & $\begin{array}{l}\text { Personas condenadas a pena de prisión de hasta } \\
5 \text { años, con la condición de que no delincan y no } \\
\text { abandonen el tratamiento durante el plazo de } \\
\text { suspensión }\end{array}$ & Art. $80.5 \mathrm{CP}$ \\
\hline \multirow[t]{2}{*}{$\begin{array}{l}\text { Respuestas } \\
\text { específicas } \\
\text { en el sistema } \\
\text { penitenciario }\end{array}$} & Programas específicos en prisión & $\begin{array}{l}\text { Personas condenadas a penas de prisión e internas } \\
\text { en centros penitenciarios, pueden beneficiarse } \\
\text { de los siguientes programas que desarrolla la } \\
\text { administración penitenciaria: } \\
\text { Programa de prevención y educación para la salud; } \\
\text { Programa de intercambio de jeringuillas; Programa } \\
\text { de tratamiento con metadona; Programa de } \\
\text { Deshabituación y Programa de reincorporación social }\end{array}$ & $\begin{array}{l}\text { Secretaría General de } \\
\text { Instituciones } \\
\text { Penitenciarias, http://www. } \\
\text { institucionpenitenciaria. } \\
\text { es/web/portal/Reeducacion/ } \\
\text { ProgramasEspecificos/ } \\
\text { drogodependencia.html }\end{array}$ \\
\hline & $\begin{array}{l}\text { Cumplimiento de pena de prisión } \\
\text { en centro de deshabituación }\end{array}$ & $\begin{array}{l}\text { Presos clasificados en } 30 \text { de tratamiento que } \\
\text { presenten una adicción a sustancias tóxicas }\end{array}$ & $\begin{array}{l}\text { Art. } 182 \text { Reglamento } \\
\text { Penitenciario }\end{array}$ \\
\hline
\end{tabular}

Estas prácticas suponen la cooperación de profesionales con formaciones y culturas profesionales distintas con objetivos y racionalidades no siempre coincidentes, las judiciales o jurídico penales, por una parte (jueces, fiscales, abogados defensores) y las sanitarias o terapéuticas, por otra (médicos, trabajadores sociales, psicólogos, enfermeros). La investigación que se expone a continuación aborda precisamente cómo estos distintos colectivos profesionales valoran la regulación y la aplicación de las respuestas penales a la drogodependencia alternativas a la prisión.

Así pues, con la hipótesis de que la pertenencia del profesional al ámbito legal o al ámbito sanitario influye en su valoración de la adecuación de las medidas penales alternativas dirigidas a abordar la delincuencia cometida por drogodependientes, se planteó el proyecto IDDO-Europe (Illicit drugs and drug offences - new challenges and developments for European criminal law politics) (Soyer y Schumann, 2015), realizado en Austria, Polonia y España, con el objetivo de evaluar las opiniones de los profesionales de los ámbitos legal y sanitario sobre algunos aspectos de la legislación relacionada con las drogas y su implementación en la práctica. En este artículo se presentan los principales hallazgos del estudio realizado en España.

\section{Materiales y métodos}

La muestra de esta investigación fue de 230 profesionales en contacto directo con consumidores de sustancias de abuso ilegales, tanto del ámbito legal (fiscales, jueces, abogados, policías) como del sanitario (en centros de atención en drogodependencias: psiquiatras, psicólogos, enfermeros y trabajadores sociales), de las ciudades de Barcelona, Granada y Bilbao. Todos ellos contestaron un cuestionario autoadministrado de respuesta múltiple diseñado ad hoc (Soyer y Schumann, 2015) que incluía (a) datos sociodemográficos y laborales: edad, sexo, profesión, cargo ocupado en la institución donde trabaja, años de experiencia en el ámbito de la dependencia de sustancias, porcentaje de dedicación de tiempo laboral a temas relacionados con delincuencia y consumo de drogas; (b) opinión sobre la respuesta a la delincuencia relacionada con drogas en la práctica, en concreto, factores que favorecen u obstaculizan la imposición MPA, tipo de sustancias que favorecen u obstaculizan la imposición de MPA, tipología de delitos que facilitan la imposición de una MPA, respuesta a la reincidencia delictiva; nivel y voluntad de cooperación existente entre profesionales de ámbito legal y sanitario; nivel de calidad de los centros aten- 
ción a las drogodependencias; (c) opinión de los profesionales en relación con la política en materia de drogas: valoración del castigo del autoconsumo de drogas; utilidad de las MPA en la prevención de la actividad delictiva; idoneidad de la actual regulación de las MPA; valoración del grado de adecuación de la aplicación de las MPA a infractores drogodependientes; utilidad de la despenalización de sustancias tipo cannabis; adecuación de tratamientos sustitutivos con agonistas opioides y valoración de la normativa vigente en materia de drogas en España. El proyecto fue aprobado por el Comité Ético de Investigación Clínica del Parc de Salut MAR (201 114420/1).

Para los análisis estadísticos se utilizó el software SPSS (versión 17.0). Se calcularon la media y desviación estándar (DE) para las variables demográficas continuas y las frecuencias para las variables discretas. Se calcularon las diferencias en los diferente ítems encuestados según tipo de profesión mediante pruebas de Chi cuadrado. Se consideró significativo cuando $\mathrm{P}>0.005$ en el chi cuadrado .

\section{Resultados}

\section{Características sociodemográficas y laborales}

La edad media de los 230 entrevistados fue de 43 años (DS 9.2; rango 21-65) y 122 (53\%) eran mujeres. La categoría profesional se distribuyó de la siguiente forma: 69 (32\%) del ámbito legal (21 jueces, 21 abogados, 19 fiscales y 8 policías) y 161 (68\%) del ámbito sanitario (71 enfermeros, 59 médicos, 16 psicólogos, 15 trabajadores sociales). La mayoría de los profesionales, un $73 \%$, trabajaba en contacto directo con personas con problemas legales relacionados con la adicción a sustancias, mientras sólo el 27\% tenía cargos de gestión. El análisis por subgrupos no mostró diferencias significativas en la distribución de sexo, excepto el grupo de agentes de policía ( $100 \%$ varones) y el grupo de enfermería, donde prevalecía el sexo femenino (65\%). El $24 \%$ de profesionales encuestados tenía una experiencia específica en el ámbito de la atención a problemas relacionados con la dependencia de sustancias de menos de 5 años, el $30 \%$ llevaba trabajando en este sector entre 5 y 10 años, el $25 \%$ de 10 a 20 años, y finalmente el $15 \%$ tenía una experiencia de más de 20 años.

\section{Comparación entre profesionales del ámbito legal y sanitario}

\section{Opinión sobre la respuesta a la delincuencia relacionada con drogas en la práctica.}

Los factores y las sustancias de abuso que favorecen $u$ obstaculizan la imposición de MPA y la valoración del grado de adecuación de la aplicación de las MPA a infractores drogodependientes, según los dos grupos de profesionales se describen en la tabla 1. En cuanto a factores a favor de la imposición de MPA, tanto los profesionales del ámbito legal como los del ámbito sanitario, coincidían que tener un entorno social estable y la situación laboral activa, son factores a favor de la imposición de MPA. Sin embargo se encontraron diferencias en cuanto a la relevancia de que se trate de un primer delito o de una primera MPA, otorgando los profesionales legales mayor relevancia al hecho de que se trate de un primer delito y que la persona no haya sido castigada antes con una MPA (Tabla 1).

Por otra parte, la mayoría de los profesionales coincidían en considerar que la reincidencia delictiva y carecer de un entorno social sólido, son factores en contra de la aplicación de las MPA; aunque diferían en cuanto a la situación de falta de ingresos económicos o de tener antecedentes de haber tenido MPA previas, puesto que los profesionales legales consideran que el desempleo y una MPA previa dificultan la aplicación de las MPA (Tabla 1).

En relación con el tipo de sustancia implicada, la mayoría de los profesionales del ámbito legal consideraron que las MPA están indicadas para delincuentes con problemas de abuso de cannabis, heroína y cocaína (54-75\%), pero únicamente una minoría de ellos sostenía que fuesen adecuadas para el abordaje del consumo de anfetaminas y otras sustancias de síntesis $(39 \%)$. Por el contrario, para los profesionales sanitarios, no había diferencias entre las sustancias, considerando que las MPA pueden estar indicadas para todas ellas (65-76\%).

La mayoría de los entrevistados considera que la aplicación de la MPA depende del tipo de delito cometido, siendo los delitos de posesión/consumo y los delitos contra la propiedad los que en mayor medida serían tributarios de MPA. Los delitos violentos son los considerados como los menos indicados para MPA aunque se observan diferencias entre los dos grupos de profesionales, siendo los profesionales legales los menos inclinados a aplicar una MPA en estos casos (Tabla 1). Así mismo, la mayoría de profesionales de ambos grupos (90 y $72 \%$ respectivamente) opina que la reacción más frecuente a la reincidencia es la aplicación de una medida más severa, mientras que únicamente un $3 \%$ y un $8 \%$ de estos profesionales considera que puede aplicar de nuevo una MPA.

La mayoría de los profesionales abogan por una mayor cooperación entre ambos ámbitos de actuación, considerando insuficiente la situación actual, tanto a nivel de la cooperación existente como de la voluntad de cooperación en ambos colectivos (Tabla1).

\section{Opinión sobre política en materia de drogas.}

No se encontraron diferencias sustanciales entre las respuestas de ambos grupos de profesionales a las preguntas sobre distintos aspectos de la política española actual en materia de drogas. Así, respecto a la opinión sobre la utilidad de castigar el consumo personal, destacó que sólo una tercera parte de los encuestados pensaban que podía ser una medi- 
Tabla 1. Opiniones de los entrevistados sobre la respuesta a la delincuencia relacionada con las drogas según su ámbito de ejercicio. España 2015.

\begin{tabular}{|c|c|c|c|c|}
\hline & $\begin{array}{c}\text { Legal } \\
(\%)\end{array}$ & $\begin{array}{c}\text { Sanitario } \\
\text { (\%) }\end{array}$ & $\mathbf{x} 2$ & $\mathbf{p}$ \\
\hline \multicolumn{5}{|c|}{ Factores a favor de la imposición de MPA } \\
\hline Entorno social estable & 68 & 73 & 0,638 & ns \\
\hline Situación laboral activa & 58 & 62 & 0,250 & ns \\
\hline Dependencia de sustancias & 44 & 42 & 0,069 & ns \\
\hline Primer delito & 75 & 58 & 6,005 & 0,014 \\
\hline Primera MPA & 67 & 33 & 22,438 & 0,000 \\
\hline \multicolumn{5}{|c|}{ Factores en contra de la imposición de MPA } \\
\hline Entorno social frágil & 60 & 62 & 0,087 & ns \\
\hline Situación de desempleo & 1 & 26 & 19,288 & 0,000 \\
\hline Dependencia de sustancias & 23 & 32 & 1,686 & ns \\
\hline Reincidencia delictiva & 77 & 80 & 0,321 & ns \\
\hline MPA previas & 52 & 38 & 4,042 & 0,044 \\
\hline \multicolumn{5}{|c|}{ Sustancias que facilitan la imposición de MPA } \\
\hline Cannabis & 75 & 76 & 0,028 & ns \\
\hline Heroína & 65 & 70 & 0,421 & ns \\
\hline Cocaína/crack & 54 & 66 & 3,064 & ns \\
\hline Anfetamina/otra & 39 & 65 & 12,777 & 0,000 \\
\hline \multicolumn{5}{|c|}{ Sustancias que obstaculizan la imposición de MPA } \\
\hline Cannabis & 29 & 24 & 0,742 & ns \\
\hline Heroína & 25 & 26 & 0,018 & ns \\
\hline Cocaína/crack & 33 & 27 & 0,843 & ns \\
\hline Anfetamina/otra & 48 & 22 & 14,916 & 0,000 \\
\hline \multicolumn{5}{|c|}{ Tipología de delitos que facilitan la imposición de una MPA } \\
\hline Posesión/consumo & 73 & 79 & 1,097 & ns \\
\hline Tráfico & 42 & 40 & 0,104 & ns \\
\hline Delitos vs propiedad & 70 & 52 & 6,087 & ns \\
\hline Crímenes violentos & 3 & 15 & 7,298 & 0,007 \\
\hline \multicolumn{5}{|c|}{ Respuesta a reincidencia delictiva } \\
\hline Nueva MPA & 3 & 8 & & \\
\hline MPA+ sanción & 7 & 20 & 10,5 & 0,005 \\
\hline Sanción más severa & 90 & 72 & & \\
\hline \multicolumn{5}{|c|}{ Nivel de cooperación existente entre profesionales de ámbito legal y sanitario } \\
\hline Suficiente & 23 & 8 & & \\
\hline Insuficiente & 64 & 76 & 8,597 & 0,014 \\
\hline Inexistente & 13 & 16 & & \\
\hline \multicolumn{5}{|c|}{ Voluntad de cooperación entre profesionales de ámbito legal y sanitario } \\
\hline Suficiente & 41 & 18 & & \\
\hline Insuficiente & 52 & 72 & 12,721 & 0,002 \\
\hline Inexistente & 7 & 10 & & \\
\hline \multicolumn{5}{|c|}{ Nivel de calidad suficiente de los centros de atención a las drogodependencias } \\
\hline Sí & 58 & 54 & 61 & ns \\
\hline
\end{tabular}

Nota. MPA: Medidas Penales Alternativas.

da útil para prevenir posteriores consumos, para prevenir el consumo de otras personas, o para reducir los delitos relacionados con la droga. Por lo que se refiere a la valoración de la efectividad de las MPA para prevenir la reincidencia, la gran mayoría de los profesionales (un $97 \%$ de los profesionales legales y de los sanitarios) consideraron que podía prevenir la delincuencia. Respecto a la valoración del actual sistema legal español sobre MPA, la mayoría de los profesionales ambos ámbitos opinaron que no es adecuada, y que habría que aplicar con más frecuencia las MPA (Tabla 2).
En cuanto a la regulación del consumo de cannabis en nuestro país, alrededor de la mitad de los profesionales tanto del ámbito legal como del sanitario, estaban de acuerdo con la situación legal actual, y sólo una minoría (aproximadamente el $10 \%$ de ellos), opinó que la ley actual debería endurecerse.

Finalmente sólo el 15\% de los profesionales del grupo legal y el 17\% de los del grupo sanitario se mostraron satisfechos con las políticas de drogas actuales. 
Tabla 2. Opiniones de los entrevistados sobre la política en materia de drogas según su ámbito de ejercicio. España 2015.

\begin{tabular}{|c|c|c|c|c|}
\hline & $\begin{array}{c}\text { Legal } \\
(\%)\end{array}$ & $\begin{array}{c}\text { Sanitario } \\
(\%)\end{array}$ & $\mathbf{X} 2$ & $\mathbf{p}$ \\
\hline \multicolumn{5}{|l|}{ Valoración del castigo autoconsumo de drogas } \\
\hline Adecuado prevención ulterior uso & 38 & 39 & 0,023 & ns \\
\hline Adecuado prevención uso por parte de otros & 35 & 31 & 0,275 & ns \\
\hline Contribuye a reducción delitos de droga & 33 & 29 & 0,481 & ns \\
\hline \multicolumn{5}{|l|}{ Utilidad de MPA para prevenir la delincuencia } \\
\hline Sí, siempre & 10 & 7 & \multirow{3}{*}{0,58} & \multirow{3}{*}{ ns } \\
\hline Sí a veces & 87 & 90 & & \\
\hline No, nunca & 3 & 3 & & \\
\hline \multicolumn{5}{|l|}{ Adecuación de la legislación actual sobre MPA } \\
\hline Sí & 16 & 10 & 1,969 & ns \\
\hline \multicolumn{5}{|l|}{ La imposición de MPA debería } \\
\hline Aumentarse & 75 & 72 & \multirow{4}{*}{1,215} & \multirow{4}{*}{ ns } \\
\hline Limitarse & 13 & 13 & & \\
\hline Mantenerse & 12 & 14 & & \\
\hline Abolirse & 0 & 1 & & \\
\hline \multicolumn{5}{|l|}{ Utilidad de la despenalización cannabis } \\
\hline Sí, para uso personal & 24 & 34 & \multirow{4}{*}{2,795} & \multirow{4}{*}{ ns } \\
\hline Sí, para comercialización & 16 & 14 & & \\
\hline No, ley actual adecuada & 50 & 41 & & \\
\hline No, ley debería endurecerse & 10 & 11 & & \\
\hline \multicolumn{5}{|c|}{ Idoneidad de los programa de mantenimiento con agonistas opioides de larga duración } \\
\hline Sí & 84 & 82 & 1,32 & ns \\
\hline \multicolumn{5}{|c|}{ Valoración de normativa vigente en política de drogas } \\
\hline Sí & 15 & 17 & 0,116 & $\mathrm{~ns}$ \\
\hline
\end{tabular}

Nota. MPA: Medidas Penales Alternativas.

\section{Opinión sobre tratamientos de las adicciones.}

La encuesta incluyó dos preguntas sobre aspectos del tratamiento actualmente ofertado en las adicciones. En este sentido, tanto los profesionales del ámbito legal como los sanitarios mostraron una relativa satisfacción sobre la calidad de los centros de atención a las adicciones, estando satisfechos del nivel de calidad de los centros un 58 y $54 \%$ de ellos, respectivamente. A su vez, más del $80 \%$ se mostró conforme con los programas actuales de mantenimiento con agonistas opioides de larga duración.

\section{Discusión}

La evaluación de las opiniones de los profesionales de ámbito legal y sanitario sobre la legislación española actual relacionada con el consumo de drogas o delitos relacionados con las mismas evidencia, en primer lugar, la coincidencia en las opiniones de ambos colectivos en las cuestiones del estudio.

Respecto a la aplicación de las MPA, los profesionales sanitarios dan menor relevancia al hecho de que se trate de un primer delito o no, y de que ya se hayan aplicado MPA previas. En este sentido, el reconocimiento de la adicción como enfermedad crónica y recidivante por parte de los profesionales sanitarios, posiblemente interviene en su actitud menos punitiva de la reincidencia delictiva relacionada con recaídas en el consumo. También destaca que para los profesionales sanitarios la decisión de imponer una MPA no depende del tipo de sustancia, mientras que los del ámbito legal relacionan más la aplicación de MPA a delitos relacionados con el consumo de heroína, cocaína o cannabis y menos en el caso de las anfetaminas $u$ otras drogas de síntesis. Probablemente esto es un reflejo de la percepción errónea de que las anfetaminas y otras drogas de síntesis son sustancias que no presentan problemas de adicción análogos a los que sí presentan otras sustancias como la heroína, cocaína y cannabis. En este sentido cabe apuntar que la demanda de tratamiento por anfetaminas y otras drogas de síntesis es mucho menor que por heroína, cocaína y cannabis (Delegación del Gobierno para el Plan Nacional sobre Drogas, 2014). Así mismo, ambos grupos de profesionales coinciden en la dificultad de aplicar una MPA en caso de delitos violentos, y reconocen como delitos apropiados para la imposición de MPA no sólo los de consumo y posesión, sino también los delitos contra la propiedad. También la mayoría de los entrevistados consideran posible prevenir la reincidencia, y apuestan por una ampliación en el ámbito de la aplicación de las MPAs. Estos resultados sugieren que los profesionales valoran la eficacia de un sistema legal, como el español, fundamentado en el reconocimiento de las adicciones como enfermedades, y en la consecuente promoción de las MPA frente a la prisión, como herramienta clave en el proceso de abordaje de los delitos relacionados con drogas, apostando por una inte- 
rrelación más sólida entre los ámbitos legal y de tratamiento de las adicciones.

Un resultado particularmente relevante del estudio es la opinión de los entrevistados en la cuestión de la despenalización del cannabis. Así, la mayoría se manifiesta contra el castigo del consumo personal, explicitando la utilidad de la despenalización del autoconsumo de cannabis, tal como se regula actualmente en España. Este dato se confirma de forma independiente en cuanto sólo una minoría de los entrevistados preferiría una normativa más severa. Dada la elevada actualidad de la discusión a nivel internacional sobre la despenalización-regulación del consumo de cannabis, esta opinión coincidente de los profesionales tanto del ámbito legal como del sanitario de la situación del consumidor de cannabis en España (Babin-Vich, 2013), puede aportar información interesante y relevante para otros países (Banys, 2016; Volkov et al., 2016; Wall et al., 2016).

Por otra parte, teniendo en cuenta que España es uno de los países con mayor cobertura de tratamientos de mantenimiento con agonistas opioides, incluyendo su disponibilidad en prisiones (Torrens, Fonseca, Castillo, Domingo-Salvany., 2013), el hecho de que el $80 \%$ de los profesionales entrevistados se mostrase conforme con las características de los programas de mantenimiento con agonistas opioides disponibles en nuestro país, parece apoyar también esta visión más orientada a considerar al consumidor como un paciente.

Finalmente, ambos grupos consideran que la cooperación entre profesionales es insuficiente, pero los profesionales del ámbito sanitario en mayor medida que los del ámbito legal. Esta percepción de la falta de cooperación puede deberse a que en la práctica, en términos generales, los profesionales del sistema legal se comunican con los sanitarios no de forma directa, sino a través de la figura de los trabajadores sociales, quienes representan un grupo minoritario dentro del grupo de ámbito sanitario. Por el contrario, el hecho de que los intermediarios en la comunicación no sean los propios profesionales sanitarios directamente responsables del caso clínico, facilita la independencia de decisiones médicas respecto a la situación legal.

Cuando comparamos nuestros resultados con los datos obtenidos mediante la misma metodología en Austria y Polonia, en el marco del proyecto IDDO-Europe, cabe destacar que los tres países tienen unas políticas legales orientadas al tratamiento, aunque España tiene una legislación más permisiva, mientras Austria y Polonia se caracterizan por una actitud más restrictiva. En los tres países se observa en general una actitud más crítica de los profesionales del ámbito sanitario hacia la efectividad de una actitud punitiva en el manejo de las adicciones, aunque en España se registra una mayor coincidencia de opinión entre los profesionales del ámbito legal y del ámbito sanitario, coincidiendo en abogar por una ulterior abertura del sistema legal vigente a las posibilidad de implementar las MPA y solicitando una ulterior revisión de las políticas vigentes sobre drogas ilegales.
La principal limitación del estudio tiene que ver con la representatividad de la muestra estudiada, que no corresponde a todo el territorio nacional.

Así pues, este estudio sobre las opiniones de la legislación actual en el campo de las adicciones a sustancias ilegales desde la perspectiva sanitaria y la legal evidencia la coincidencia de opiniones en numerosos aspectos sobre los que se ha investigado así como los principales puntos de discrepancia, ofreciendo un marco de trabajo para la mejoría de la interacción entre ambos grupos de profesionales y la mejoría final del abordaje de la enfermedad adictiva.

\section{Conflicto de intereses}

Los autores declaran la inexistencia de conflictos de intereses.

\section{Reconocimientos}

Este estudio ha sido financiado en parte por Open SocietyInstitute (OSI) en el proyecto (Illicitdrugs and drugoffences - new challenges and developmentsforEuropean criminal lawpolitics -IDDO-Europe) y por la Red de Trastornos Adictivos UE-FEDER 2012, RD12/0028/0009.

Los autores desean expresar su gratitud a Miguel Landabaso por facilitar el reclutamiento de entrevistados. Agradecemos a Nahia Zorrilla, profesora en la Universitat Pompeu Fabra, su contribución en la discusión de los resultados.

\section{Referencias}

Babín Vich, F. A. (2013). The debate over drug legalization. Adicciones, 25, 6-9.

Banys, P. (2016). Mitigation of Marijuana-Related Legal Harms to Youth in California. Journal of Psychoactive Drugs, 48, 11-20. doi:10.1080/02791072.2015.1126770.

Delegación del Gobierno para el Plan Nacional sobre Drogas (DGPNSD) (2014). Informe del Observatorio Español de la Droga y las Toxicomanías y Estadísticas OEDT (2013). Recuperado de http://www.pnsd.msssi. gob.es/profesionales/publicaciones/catalogo/catalogoPNSD/publicaciones/pdf/Informe_y_Estadisticas_ OEDT_2013.pdf.

Delegación del Gobierno para el Plan Nacional sobre Drogas (DGPNSD) (2013). Memoria. Ministerio de Sanidad, Servicios Sociales y Igualdad, Secretaría de Estado de Servicios Sociales e Igualdad. Recuperado de http://www.pnsd.msssi.gob.es/profesionales/publicaciones/catalogo/catalogoPNSD/publicaciones/pdf/ memo2013.pdf.

Esbec, E. y Echeburúa, E. (2016). Substance abuse and crime: considerations for a comprehensive forensic assessment. Adicciones, 28, 48-56. doi:10.20882/adicciones. 790 . 
European Monitoring Centre for Drugs and Drug Addiction (2007). Drugs and crime a complex relationship. Recuperado de http://www.emcdda.europa.eu/system/files/publications/470/Dif16EN_85000.pdf.

European Monitoring Centre for Drugs and Drug Addiction (2015). Alternatives to punishment for drug-using offenders. Recuperado de http://www.drugs.ie/resourcesfiles/ResearchDocs/Europe/Research/2015/EMCDDA_Alternatives_to_punishment.pdf.

Soyer, R. y Schumann, S. (Eds.) (2015). Treatment versus Punishment for Drug Addiction. Cham: Springer International Publishing.

Torrens, M., Fonseca, F., Castillo, C. y Domingo-Salvany, A. (2013). Methadone maintenance treatment in Spain: the success of a harm reduction approach. Bulletin of the World Health Organization, 91, 136-141. doi:10.2471/ BLT.12.111054.

Volkow, N. D., Swanson, J. M., Evins, A. E., DeLisi, L. E., Meier, M. H., Gonzalez, R.,... Baler, R. (2016). Effects of Cannabis Use on Human Behavior, Including Cognition, Motivation, and Psychosis: A Review. JAMA Psychiatry, 73, 292-297. doi:10.1001/jamapsychiatry.2015.3278.

Wall, M. M., Mauro, C., Hasin, D. S., Keyes, K. M., Cerda, M., Martins, S. S. y Feng, T. (2016). Prevalence of marijuana use does not differentially increase among youth after states pass medical marijuana laws: Commentary on and reanalysis of US National Survey on Drug Use in Households data 2002-2011. The International Journal on Drug Policy, 29, 9-13. doi:10.1016/j.drugpo.2016.01.015. 